Bull. Austral. Math. Soc.

$16 \mathrm{~A} 68,16 \mathrm{~A} 72,16 \mathrm{~A} 12$

VOL. 44 (1991) [233-238]

\title{
JORDAN MAPPINGS OF SEMIPRIME RINGS II
}

\author{
MAteJ Brešar
}

We describe Jordan homomorphisms and Jordan triple homomorphisms onto 2torsion free semiprime rings in which the annihilator of any ideal is a direct summand.

\section{INTRODUCTION AND PRELIMINARIES}

The aim of this paper is to complete some results from our work [3], concerned with Jordan homomorphisms (that is, additive mappings of rings satisfying $\theta(a b+b a)=$ $\theta(a) \theta(b)+\theta(b) \theta(a)$ ) and Jordan triple homomorphisms (that is, additive mappings satisfying $\theta(a b a)=\theta(a) \theta(b) \theta(a))$. First, let us look at two simple examples of Jordan homomorphisms.

EXAMPLE 1: Let $R, U^{\prime}$ and $V^{\prime}$ be rings, and let $\varphi: R \rightarrow U^{\prime}$ and $\psi: R \rightarrow V^{\prime}$ be a homomorphism and an antihomomorphism, respectively. Define $\theta: R \rightarrow U^{\prime} \oplus V^{\prime}$ by $\theta(r)=(\varphi(r), \psi(r))$. Then $\theta$ is a Jordan homomorphism.

The next example is, in fact, the special case of Example 1.

ExAmple 2: Let $U, V, U^{\prime}$ and $V^{\prime}$ be rings, and let $\varphi: U \rightarrow U^{\prime}$ and $\psi: V \rightarrow$ $V^{\prime}$ be a homomorphism and an antihomomorphism, respectively. Then the mapping $\theta: U \oplus V \rightarrow U^{\prime} \oplus V^{\prime}, \theta(u, v)=(\varphi(u), \psi(v))$, is a Jordan homomorphism.

Note the important difference: in Example 2 the image of $\theta$ is an (associative) subring, while in Example 1 this need not be true.

Let $\theta$ be a Jordan homomorphism of a ring $R$ onto a 2 -torsion free semiprime ring $R^{\prime}$. Baxter and Martindale showed that in this case there exists an essential ideal $E$ of $R$ such that the restriction of $\theta$ to $E$ is of the same form as the mapping $\theta$ in Example 1 [2, Theorem 2.7]. Roughly speaking, in [3, Theorem 2.3] we generalised their result by showing that this restriction is rather of the form as in Example 2. Baxter and Martindale also proved that if $R^{\prime}$ is centrally closed (that is, its centroid coincides with its extended centroid) then the restriction to an essential ideal is unnecessary, thus $\theta$ is like in Example 1 [2, Theorem 3.8]. In this paper we show that in the case of centrally closed semiprime rings $\theta$ is rather like in Example 2. In fact, we prove that this is

Received 21 September 1990

Copyright Clearance Centre, Inc. Serial-fee code: 0004-9729/91 \$A2.00+0.00. 
true for a more general class of semiprime rings, that is, semiprime rings in which the annihilator of any ideal is a direct summand.

Let $I$ be an ideal of a semiprime ring $R^{\prime}$. It can be easily shown that the left and right and two-sided annihilators $\operatorname{Ann}(I)$ of $I$ coincide. Next, $I \cap \operatorname{Ann}(I)=0$ and $I \oplus \operatorname{Ann}(I)$ is an essential ideal of $R^{\prime}$. As we have mentioned, we will be concerned with semiprime rings $R^{\prime}$ in which the annihilator of any ideal is a direct summand; that is, Ann $(I) \oplus \operatorname{Ann}(\operatorname{Ann}(I))=R^{\prime}$ for every ideal $I$ of $R^{\prime}$. Every prime ring trivially satisfies this condition. Moreover, the same is true for a direct sum of prime rings. Another important example is a semiprime Baer ring (see [4, Theorem 13]). Next we have

Lemma 1. Let $R^{\prime}$ be a centrally closed semiprime ring. Then the annihilator of any ideal in $R^{\prime}$ is a direct summand.

Proof: In [1] Amitsur defined the notion of a closed ideal in a semiprime ring (not to be confused with the notion of a centrally closed semiprime ring): an ideal $U$ is closed if $U=\operatorname{Ann}(\operatorname{Ann}(U))$. We claim that the ideal $U$ in any semiprime ring is closed if and only if it is the annihilator of some ideal. If $U$ is closed then $U$ is the annihilator of the ideal Ann $(U)$. Conversely, let $U$ be the annihilator of an ideal $V$. Then $V \subseteq \operatorname{Ann}(U)$ and so $\operatorname{Ann}(\operatorname{Ann}(U)) \subseteq \operatorname{Ann}(V)=U$; hence $U$ is closed.

Now, let $R^{\prime}$ be a centrally closed semiprime ring, and let $J$ be the annihilator of some ideal of $R^{\prime}$. We want to show that $J$ is a direct summand. By the above argument $J$ is a closed ideal and so it follows from [1, Corollary 9] that $J=R^{\prime} \cap Q_{0} \varepsilon$ where $Q_{0}$ is the ring of quotients of $R^{\prime}$ and $\varepsilon$ is an idempotent contained in the extended centroid of $R^{\prime}$ (that is, the centre of $Q_{0}$ ). However, $R^{\prime}$ is centrally closed and so $\varepsilon$ actually lies in the centroid of $R^{\prime}$, thus $R^{\prime} \varepsilon \subseteq R^{\prime}$. We claim that $J=R^{\prime} \varepsilon$. Clearly $R^{\prime} \varepsilon \subseteq J$. Conversely, since $J \subseteq Q_{0} \varepsilon$ we have $x=x \varepsilon$ for every $x \in J$, and therefore $J \subseteq R^{\prime} \varepsilon$. Of course, $R^{\prime}=R^{\prime} \varepsilon \oplus R^{\prime}(1-\varepsilon)$ which means that $J=R^{\prime} \varepsilon$ is indeed a direct summand.

\section{The Results}

Our first theorem is an extension of Theorem 2.3 in [3]. Fortunately the same proof works, but we include it for the sake of completeness.

Theorem 1. Let $\theta$ be a Jordan homomorphism of a ring $R$ onto a 2-torsion free semiprime ring $R^{\prime}$ in which the annihilator of every ideal is a direct summand. Then there exist ideals $U$ and $V$ of $R$ and ideals $U^{\prime}$ and $V^{\prime}$ of $R^{\prime}$ such that $U \cap V=\operatorname{Ker} \theta$ and $U+V=R, U^{\prime} \cap V^{\prime}=0$ and $U^{\prime} \oplus V^{\prime}=R^{\prime}$, the restriction of $\theta$ to $U$ is a homomorphism of $U$ onto $U^{\prime}$, and the restriction of $\theta$ to $V$ is an antihomomorphism of $V$ onto $V^{\prime}$. 
ProOF: We introduce the abbreviations $a^{b}=\theta(a b)-\theta(a) \theta(b), a_{b}=\theta(a b)-$ $\theta(b) \theta(a)$. By [3, Corollary 2.2] we have

$$
a^{b} R^{\prime} c_{d}=0 \quad \text { for all } \quad a, b, c, d \in R .
$$

Let $V_{0}^{\prime}$ be an ideal of $R^{\prime}$ generated by the set $\left\{a^{b} \mid a, b \in R\right\}$, and set $U^{\prime}=\operatorname{Ann}\left(V_{0}^{\prime}\right)$, $V^{\prime}=\operatorname{Ann}\left(U^{\prime}\right)$. By assumption, $U^{\prime} \oplus V^{\prime}=R^{\prime}$. Of course, $\left\{a^{b} \mid a, b \in R\right\} \subseteq V_{0}^{\prime} \subseteq V^{\prime}$, and from (1) we see that $\left\{a_{b} \mid a, b \in R\right\} \subseteq U^{\prime}$. Let $U=\theta^{-1}\left(U^{\prime}\right)$ and $V=\theta^{-1}\left(V^{\prime}\right)$. Take $u \in U, y \in R, x^{\prime} \in R^{\prime}$. By (1) we have

$$
\begin{aligned}
u^{y} x^{\prime} u^{y} & =u^{y} x^{\prime}\left(u^{y}-u_{y}\right) \\
& =u^{y} x^{\prime}(\theta(y) \theta(u)-\theta(u) \theta(y)) \\
& =u^{y} x^{\prime} \theta(y) \theta(u)-\left(u^{y} x^{\prime} \theta(u)\right) \theta(y) \\
& =0
\end{aligned}
$$

since $\theta(u) \in U^{\prime}=\operatorname{Ann}\left(V_{0}^{\prime}\right)$. Hence $u^{y}=0$ by the semiprimeness of $R^{\prime}$, that is, $\theta(u y)=\theta(u) \theta(y)$ for all $u \in U, y \in R$. By the definition of Jordan homomorphisms we then also have $\theta(y u)=\theta(y) \theta(u)$. The last two relations imply that $U$ is an ideal of $R^{\prime}$. Clearly $\theta(U)=U^{\prime}$. Thus we have proved that the restriction of $\theta$ to $U$ is a homomorphism of $U$ onto $U^{\prime}$. In a similar fashion one shows that $V$ is an ideal of $R$, and that the restriction of $\theta$ to $V$ is an antihomomorphism of $V$ onto $V^{\prime}$.

It is obvious that $\operatorname{Ker} \theta \subseteq U \cap V$. Conversely, $\theta(U \cap V) \subseteq \theta(U) \cap \theta(V)=0$ and therefore $U \cap V=\operatorname{Ker} \theta$. Let us show that $U+V=R$. Given $x \in R$, we have $\theta(x)=u^{\prime}+v^{\prime}$ where $u^{\prime} \in U^{\prime}, v^{\prime} \in V^{\prime}$. Since $u^{\prime}=\theta(u), u \in U$, and $v^{\prime}=\theta(v), v \in V$, it follows that $x-u-v \in \operatorname{Ker} \theta=U \cap V$. Hence $U+V=R$. The proof of the theorem is complete.

REMARK. Combining Theorem 1 and Lemma 1 we obtain, as outlined in the introduction, a generalisation of Theorem 3.8 in [2]. Note also that Theorem 3.9 in [2] can now be stated in a more general form.

We now want to prove the analogous result for Jordan triple homomorphisms. The proof is an adaption of the proof of Theorem 3.5 in [3].

Theorem 2. Let $R$ be a ring with property $R^{2}=R$, and let $R^{\prime}$ be a 2-torsion free semiprime ring in which the annihilator of any ideal is a direct summand. If $\theta$ is a Jordan triple homomorphism of $R$ onto $R^{\prime}$ then there exist ideals $U_{1}, U_{2}, U_{3}, U_{4}$ of $R$, and ideals $U_{1}^{\prime}, U_{2}^{\prime}, U_{3}^{\prime}, U_{4}^{\prime}$ of $R^{\prime}$ such that

(i) $U_{i} \cap U_{j}=\operatorname{Ker} \theta, i \neq j$, and $U_{1}+U_{2}+U_{3}+U_{4}=R$,

(ii) $U_{i}^{\prime} \cap U_{j}^{\prime}=0, i \neq j$, and $U_{1}^{\prime} \oplus U_{2}^{\prime} \oplus U_{3}^{\prime} \oplus U_{4}^{\prime}=R^{\prime}$,

(iii) the restriction of $\theta$ to $U_{1}$ is a homomorphism of $U_{1}$ onto $U_{1}^{\prime}$, 
(iv) the restriction of $\theta$ to $U_{2}$ is a negative of a homomorphism of $U_{2}$ onto $U_{2}^{\prime}$,

(v) the restriction of $\theta$ to $U_{3}$ is an antihomomorphism of $U_{3}$ onto $U_{3}^{\prime}$,

(vi) the restriction of $\theta$ to $U_{4}$ is a negative of an antihomomorphism of $U_{4}$ onto $U_{4}^{\prime}$.

Proof: We write $S(a, b, c)=\theta(a b c)-\theta(a) \theta(b) \theta(c)$ and $T(a, b, c)=\theta(a b c)-$ $\theta(c) \theta(b) \theta(a)$. By $[3$, Corollary 3.2$]$ we have

$$
T\left(a_{1}, a_{2}, a_{3}\right) R^{\prime} S\left(b_{1}, b_{2}, b_{3}\right)=0 \quad \text { for all } a_{i}, b_{i} \in R, \quad i=1,2,3 .
$$

Let $I_{0}^{\prime}$ be an ideal of $R^{\prime}$ generated by the set $\{S(a, b, c) \mid a, b, c \in R\}$ and let $U^{\prime}=$ $\operatorname{Ann}\left(I_{0}^{\prime}\right), V^{\prime}=\operatorname{Ann}\left(U^{\prime}\right)$. By assumption, $U^{\prime} \oplus V^{\prime}=R^{\prime}$. Of course, $\{S(a, b, c)\}$ $a, b, c \in R\} \subseteq V^{\prime}$, and by (2) we have $\{T(a, b, c) \mid a, b, c \in R\} \subseteq U^{\prime}$. We set $U=\theta^{-1}\left(U^{\prime}\right)$ and $V=\theta^{-1}\left(V^{\prime}\right)$. Take $x, y, x \in R$ with at least one in $U$. According to (2) for any $x^{\prime} \in R^{\prime}$ we have

$$
\begin{aligned}
& S(x, y, z) x^{\prime} S(x, y, z) \\
& \quad=S(x, y, z) x^{\prime}(S(x, y, z)-T(x, y, z)) \\
& \quad=S(x, y, z) x^{\prime}(\theta(z) \theta(y) \theta(x)-\theta(x) \theta(y) \theta(z)) \\
& \quad=0
\end{aligned}
$$

since at least one of $\theta(x), \theta(y), \theta(z)$ lies in $U^{\prime}$. Consequently $S(x, y, z)=0$. Since $R^{2}=R$ we have $\theta(U R)=\theta(U R R)=\theta(U) \theta(R) \theta(R) \subseteq U^{\prime}$ which means that $U$ is a right ideal of $R$. Similarly we see that $U$ is a left ideal.

Analogously one shows that $V$ is an ideal of $R$ and that $T(v, x, y)=T(x, v, y)=$ $T(x, y, v)=0$ holds for all $x, y \in R, v \in V$.

Consider $\theta(u x y u x)$ where $x, y \in R$ and $u \in U$. On the one hand we have

$$
\begin{aligned}
\theta(u x y u x) & =\theta(u(x y u) x) \\
& =\theta(u) \theta(x y u) \theta(x) \\
& =\theta(u) \theta(x) \theta(y) \theta(u) \theta(x)
\end{aligned}
$$

and on the other hand,

$$
\begin{aligned}
\theta(u x y u x) & =\theta((u x) y(u x)) \\
& =\theta(u x) \theta(y) \theta(u x) .
\end{aligned}
$$

Comparing the last two relations we arrive at $P(u, x) \theta(y) Q(u, y)+Q(u, x) \theta(y) P(u, x)=$ 0 where $P(r, s)$ denotes the element $\theta(r s)-\theta(r) \theta(s)$, and $Q(r, s)$ denotes $\theta(r s)+$ 
$\theta(r) \theta(s)$. Since $\theta$ is onto it follows from [3, Lemma 1.1] that $P(u, x) R^{\prime} Q(u, x)=0$ for all $u \in U, x \in R$. But then [3, Lemma 1.2] tells us that

$$
P(u, x) R^{\prime} Q(w, y)=0 \text { for all } u, w \in U, x, y \in R .
$$

Let $I_{1}^{\prime}$ be an ideal of $R^{\prime}$ generated by the set $\{P(u, x) \mid u \in U, x \in R\}$. Since $U$ is an ideal we see that $P(u, x)$ and $Q(u, x)$ lie in $U^{\prime}$ if $u \in U$. Therefore $I_{1}^{\prime}$ is contained in $U^{\prime}$. Let $U_{1}^{\prime}=\operatorname{Ann}\left(I_{1}^{\prime}\right) \cap U^{\prime}, U_{2}^{\prime}=\operatorname{Ann}\left(U_{1}^{\prime}\right) \cap U^{\prime}$.

We claim that $U_{1}^{\prime} \oplus U_{2}^{\prime}=U^{\prime}$. Clearly $U_{1}^{\prime} \cap U_{2}^{\prime}=0$. By assumption, Ann $\left(I_{1}^{\prime}\right)$ is a direct summand, thus $R^{\prime}=\operatorname{Ann}\left(I_{1}^{\prime}\right) \oplus W^{\prime}$ for some ideal $W^{\prime}$ of $R^{\prime}$. Then $W^{\prime}=\operatorname{Ann}\left(\operatorname{Ann}\left(I_{1}^{\prime}\right)\right) \subseteq \operatorname{Ann}\left(\operatorname{Ann}\left(I_{1}^{\prime}\right) \cap U^{\prime}\right)=\operatorname{Ann}\left(U_{1}^{\prime}\right)$, and, since $I_{1}^{\prime} \subseteq U^{\prime}$, $W^{\prime}=\operatorname{Ann}\left(\operatorname{Ann}\left(I_{1}^{\prime}\right)\right) \subseteq \operatorname{Ann}\left(\operatorname{Ann}\left(U^{\prime}\right)\right)=\operatorname{Ann}\left(V^{\prime}\right)=U^{\prime}$. Thus $W^{\prime} \subseteq U_{2}^{\prime}$ (in fact, $\left.W^{\prime}=U_{2}^{\prime}\right)$. Given $u^{\prime} \in U^{\prime}$, we then have $u^{\prime}=z^{\prime}+w^{\prime}$ for some $z^{\prime} \in \operatorname{Ann}\left(I_{1}^{\prime}\right), w^{\prime} \in W^{\prime}$. Since $W^{\prime} \subseteq U^{\prime}$ we then have $z^{\prime} \in U^{\prime}$; that is, $z^{\prime} \in \operatorname{Ann}\left(I_{1}^{\prime}\right) \cap U^{\prime}=U_{1}^{\prime}$. Hence $U^{\prime}=U_{1}^{\prime}+U_{2}^{\prime}$.

We set $U_{1}=\theta^{-1}\left(U_{1}^{\prime}\right)$ and $U_{2}=\theta^{-1}\left(U_{2}^{\prime}\right)$. Take $u_{1} \in U_{1}, x \in R, y^{\prime} \in R^{\prime}$. By (3) we have

$$
\begin{aligned}
P\left(u_{1}, x\right) y^{\prime} P\left(u_{1}, x\right) & =P\left(u_{1}, x\right) y^{\prime}\left(P\left(u_{1}, x\right)-Q\left(u_{1}, x\right)\right) \\
& =-2 P\left(u_{1}, x\right) y^{\prime} \theta\left(u_{1}\right) \theta(x) \\
& =0
\end{aligned}
$$

since $\theta\left(u_{1}\right) \in U_{1}^{\prime}$. Thus $P\left(u_{1}, x\right)=0$ by the semiprimeness of $R^{\prime}$. This means that the restriction of $\theta$ to $U_{1}$ is a homomorphism of $U_{1}$ onto $U_{1}^{\prime}$. The last relation also implies that $U_{1}$ is a right ideal of $R$. In order to prove that $U_{1}$ is a left ideal we will show that $P\left(x, u_{1}\right)$ with $x \in R, u_{1} \in U_{1}$, is zero as well. Take $u_{1} \in U_{1}, x, y, z \in R$. Since $x u_{1} \in U$ we have $\theta\left(\left(x u_{1}\right) y z\right)=\theta\left(x u_{1}\right) \theta(y) \theta(z)$. But on the other hand, using $u_{1} y \in$ $U$ and $P\left(u_{1}, y\right)=0$ we obtain $\theta\left(x\left(u_{1} y\right) z\right)=\theta(x) \theta\left(u_{1} y\right) \theta(z)=\theta(x) \theta\left(u_{1}\right) \theta(y) \theta(z)$. Comparing, we arrive at $\left(\theta\left(x u_{1}\right)-\theta(x) \theta\left(u_{1}\right)\right) \theta(y) \theta(z)=0$. But then, since $\theta$ is onto and $R$ is semiprime, it follows that $\theta\left(x u_{1}\right)=\theta(x) \theta\left(u_{1}\right)$.

Note that (3) implies $\{Q(u, x) \mid u \in U, x \in R\} \subseteq U_{1}^{\prime}$. Using similar arguments as above one then verifies that $Q\left(u_{2}, x\right)=0$ and $Q\left(x, u_{2}\right)=0$ for all $u_{2} \in U_{2}, x \in R$. This implies that $U_{2}$ is an ideal of $R$ and that the restriction of $\theta$ of $U_{2}$ is a negative of a homomorphism of $U_{2}$ onto $U_{2}^{\prime}$.

In an analogous way one shows that there exist ideals $U_{3}^{\prime}, U_{4}^{\prime}$ of $R^{\prime}$ such that $U_{3}^{\prime} \oplus U_{4}^{\prime}=V^{\prime}$, and that $U_{3}=\theta^{-1}\left(U_{3}^{\prime}\right), U_{4}=\theta^{-1}\left(U_{4}^{\prime}\right)$ are ideals of $R$ satisfying $\theta\left(u_{3} x\right)=\theta(x) \theta\left(u_{3}\right), \theta\left(x u_{3}\right)=\theta\left(u_{3}\right) \theta(x), \theta\left(u_{4} x\right)=-\theta(x) \theta\left(u_{4}\right), \theta\left(x u_{4}\right)=-\theta\left(u_{4}\right) \theta(x)$ for all $x \in R, u_{3} \in U_{3}, u_{4} \in U_{4}$. Then, of course, the restriction of $\theta$ to $U_{3}$ is an antihomomorphism of $U_{3}$ onto $U_{3}^{\prime}$, and the restriction of $\theta$ to $U_{4}$ is a negative of an antihomomorphism of $U_{4}$ onto $U_{4}^{\prime}$. 
Clearly $U_{i}^{\prime} \cap U_{j}^{\prime}=0, i \neq j$, and $U_{1}^{\prime} \oplus U_{2}^{\prime} \oplus U_{3}^{\prime} \oplus U_{4}^{\prime}=R^{\prime}$. It remains to prove (i). It is obvious that $\operatorname{Ker} \theta \subseteq U_{i}$, and therefore $\theta\left(U_{i} \cap U_{j}\right) \subseteq \theta\left(U_{i}\right) \cap \theta\left(U_{j}\right)=U_{i}^{\prime} \cap U_{j}^{\prime}=0$, $i \neq j$, implies $U_{i} \cap U_{j}=\operatorname{Ker} \theta, i \neq j$. Let us show that $U+V=R$. Given $x \in R$, the element $\theta(x)$ can be written in the form $u^{\prime}+v^{\prime}, u^{\prime} \in U^{\prime}, v^{\prime} \in V^{\prime}$. There exist $u \in U, v \in V$ such that $\theta(u)=u^{\prime}$ and $\theta(v)=v^{\prime}$. Hence $x-u-v \in \operatorname{Ker} \theta=U \cap V$ which means that $U+V=R$. Similarly we verify that $U_{1}+U_{2}=U$ and $U_{3}+U_{4}=V$. Consequently $U_{1}+U_{2}+U_{3}+U_{4}=R$. The proof of the theorem is complete.

\section{ReFeRENCES}

[1] A.S. Amitsur, 'On rings of quotients', Sympos. Math. 8 (1972), 149-164.

[2] W.E. Baxter and W.S. Martindale, 'Jordan homomorphisms of semiprime rings', J. Algebra 56 (1979), 457-471.

[3] M. Brešar, 'Jordan mappings of semiprime rings', J. Algebra 127 (1989), 218-228.

[4] I. Kaplansky, Rings of operators (Benjamin, New York, 1968).

University of Maribor

PF

Korołka 160

62000 Maribor

Yugoslavia 